\title{
From Liquid Structure to Configurational Entropy: Introducing Structural Covariance
}

\author{
Pierre Ronceray \\ LPTMS, CNRS, Univ. Paris-Sud, \\ Université Paris-Saclay, 91405 Orsay, France \\ and Peter Harrowell \\ School of Chemistry, University of Sydney, Sydney N.S.W. 2006, Australia
}

\begin{abstract}
We connect the configurational entropy of a liquid to the geometrical properties of its local energy landscape, using a high-temperature expansion. It is proposed that correlations between local structures arises from their overlap and, being geometrical in nature, can be usefully determined using the inherent structures of high temperature liquids. We show quantitatively how the hightemperature covariance of these local structural fluctuations arising from their geometrical overlap, combined with their energetic stability, control the decrease of entropy with decreasing energy. We apply this formalism to a family of Favoured Local Structure (FLS) lattice models with two low symmetry FLS's which are found to either crystallize or form a glass on cooling. The covariance, crystal energy and estimated freezing temperature are tested as possible predictors of glass-forming ability in the model system.
\end{abstract}

\section{INTRODUCTION}

Why study liquid structure? The longstanding answer to this question is that structure is expected to provide a microscopic account of the experimentally measured properties of liquids. The theoretical treatment of these properties, e.g. the equation of state and the structure factor, are the goals that drove the development of liquid state theory through the 1960's and 70's [1. The structural information required for these tasks turned out to be modest. The scattering function only requires the distribution of pairwise separations and, if we can assume pairwise additive interaction potentials, so too does the equation of state 1 . Closures of correlation function hierarchies have provided reasonable radial distribution functions (at least for single component atomic liquids) without explicit reference to the geometrical structure of the liquid [1]. This bypassing of most of the liquid structure in the development of liquid theory is one of cornerstones of its successes. It does leave us, however, still casting around for a motivation to study the full many-body aspects of liquid structure.

A second reason for the study of liquid structure is related to the properties of deeply supercooled liquids. The slow relaxation of viscous liquids 2 and the rigidity of amorphous solids 3 arise as a consequence of the constraint that a configuration of the system exerts on the motion of its constituent particles at low temperature. This second goal of the study of liquid structure is, therefore, to uncover an intelligible rationale for the origin and persistence of these constraints. How structure might accomplish this goal is worth fleshing out. A structural description, here taken to mean a reduced description of a configuration in terms of some set of local structures - e.g. the coordination polyhedra - is just an exercise in descriptive geometry, with the choice of structural components left to the whim of the researcher. A geometrical analysis of a configuration of the system allows, for each of its constituent particles, to identify the specific local structure that characterizes its molecular neighbourhood among a dictionary of possible local environments. The utility of a particular structural description can be assessed by the information compression that it provides. The connection to physics arises because this information compression relies on the presence of geometrical constraints. A particular coordination polyhedron, for example, embodies a number of constraints between bond angles, numbers of nearest neighbours, topological connectivity, etc. It is these geometrical constraints that permit the information reduction in the first place. The hope that underlies this structural analysis is that these geometrical constraints on structure translate into physical constraints on particle positions. A well studied example of the fulfillment of this hope is a binary Lennard-Jones mixture introduced by Wahnström 4 for which the correlation between local icosahedral topology and slow relaxation has been well established 5. This structure $\rightarrow$ constraint program depends on the information reduction achieved by a given structural analysis. Too complex a liquid structure and the associated information compression is insufficient to result in any significant physical constraints. Many supercooled liquids exhibit structures best characterised by as a diverse range of local structures, all occurring with comparable but low frequency. In these cases, it is difficult to see how broadly diverse structure can be linked to the presence of physical constraint.

In this paper we concentrate on a third motivation for studying liquid structure, one whose utility does not depend on whether the liquid structure is simple or complex and that has received little attention in the literature. We explore how the liquid structure connects to the configurational entropy of the liquid and, hence, plays a crucial role in determining the stability of the liquid with respect to crystallization. 
The concept of a configurational entropy plays a central role in the modern description of supercooled liquids [6. The proposal is that the rough energy landscape that characterizes a supercooled liquid can be usefully described in terms of local minima of the potential energy - the inherent structures of the system - and fluctuations around these minima. The total entropy of the liquid can thus be resolved into two parts - the first one, termed configurational entropy, associated with the choice of the inherent structure, and the second with the fluctuations of particles around these metastable configurations. Computationally, the configurational entropy has been defined explicitly as $S_{c}=k_{B} \log N_{\text {is }}$ where $N_{\text {is }}$ is the number of distinct inherent structure [7. Calculations based on this definition of the configurational entropy have been carried out for binary Lennard-Jones mixtures [8], silica [9] along with other model liquids [10]. Such calculations reveal that over a wide range of temperatures, the configurational entropy can be reasonably described as a quadratic function of the potential energy:

$$
S_{c}=S_{\infty}-\frac{1}{2 K}\left(E-E_{\infty}\right)^{2}
$$

The quantities $S_{\infty}$ and $E_{\infty}$ respectively correspond to the entropy and energy of inherent structures in the high temperature limit. This expression was shown to provide a good approximation of the configurational entropy for both a binary atomic mixture [11] and a model of silica [12. Sastry, Debenedetti and Stillinger [13. have demonstrated the existence of this high-temperature limit by showing that there exists a temperature, $T_{x}$, above which the average energy of inherent structures does not depend on the temperature of the initial liquid. The idea is that with sufficient thermal energy, the liquid energy lies above the "top" of the potential energy landscape and so is able to effectively sample all possible inherent structures. Referring to the empirical expression in Equation 1, the change of liquid's thermodynamic quantities upon cooling are then controlled by the quantity $K$, which determines the entropic cost of lowering the energy.

The aim of this article is to relate the thermodynamic properties of a liquid - and in particular the value of $K$ - to its microscopic structural origins. The liquid's local structural properties will be described macroscopically in terms of the frequencies of each type of local structure. Local structures couple directly to the energy of the system: indeed, not all local environments around a particle are equivalent from an energetic point of view. For a given Hamiltonian, some local structures are particularly stable, while others are disfavoured; the energies associated to local structures constitute the local energy landscape of the system. On cooling, the liquid energy will decrease, which implies that the frequencies of lowenergy local structures will increase. On the other hand, this accumulation of stable local structures comes with an entropy cost: indeed, the structural frequencies constrain the associated size of the configuration space, and thus the configurational entropy. The geometrical properties of the local structures have a direct influence on this configurational entropy, and thus on the thermodynamic properties of the system.

In this article, we present an analysis of liquid structure that goes beyond mere description, and allows quantitative predictions that become exact at high temperature, while retaining relevant information at low temperature. We focus on the role of correlations between the frequencies of local structures arising from their geometrical overlap - the structural covariances. We show their crucial importance in connecting the local energy landscape - i.e. the energy of local structures - to the global energy landscape - characterized by the configurational entropy of the system. In Section [II we present our theoretical framework in a general context of off-lattice liquids, establishing a relationship between liquid structure and configurational entropy. While we expect our predictions to hold in such cases, we have so far only tested them in the context of a class of lattice models of liquids inspired from our previous works, the Favoured Local Structures model with two stable local structures, as presented in Section III Our goals in this Section are to provide some measure of the validity of the high temperature approximation. Employing our structural covariance framework, we present new results concerning a version of the FLS lattice model that appears to capture important aspects of the problem of glass-forming ability.

\section{LOCAL LIQUID STRUCTURES AND THEIR COVARIANCE}

In this Section, we develop a theoretical relation between local structure and configurational entropy in an off-lattice liquid. There is some assumed degree of arbitrariness in the definition of local structures, as already discussed in the Introduction, and the precise definition and choice of local structural parameter is usually adapted to the specific needs of the study. A number of choices for these structural components have been considered in the literature - Voronoi polyhedra [14, Delaunay tessellations [15], common neighbours [16], bond ring statistics [17, bond orientation order [18] and tetrahedrality [19. One of the most common is the local coordination polyhedra 20] similar to a Delaunay vertex except that the neighbours are defined by a cutoff separation rather than a geometric condition based on spacefilling. The literature regarding these various choices has been summarised in a number of excellent reviews [20]. The choice of structural components depends on the goal of the analysis and the expectation of the type of structure that might be found. Voronoi or Delaunay tilings address the question of how structure fills space. Common neighbour analysis represents a reduction to the smallest non-trivial set of structural components out of which distinct collective structures can be assembled. Tetrahedrality focuses on the structural significance of triangula- 
tion with respect to mechanical stability. It is important to remark that for all definitions of local structure, there is some degree of overlap between a local structure and its neighbours. For instance, Voronoi polyhedra share faces, while a particle contributes to the local coordination polyhedra of all its neighbours. This implies that the local structure at a point imposes geometrical constraints on the surrounding local structures. The overlap of coordination polyhedra was the basis of the geometrical construction used by Frank and Kasper in their classic papers on tetrahedral solids [21.

In the analysis presented below, we consider only the inherent structures of a liquid. These are the configurations associated with local minima of the potential energy. We assume that in such states, the environment of each particle is well characterized by its local structure, and that the total potential energy can be written as a sum of the energies of the individual local structures. This assumption implies that the system can be fully described in terms of its local energy landscape, i.e. the local energy associated with possible structural components. The coordination polyhedra about each particle (in concert with an assumed short range interaction) represents a good realization of these requirements and, for concreteness, we will select it as our definition for local structural components in the following.

\section{A. The Structure and Energy Vectors}

Having chosen a definition of local structures, we can establish a dictionary of all possibilities for such local structures, which we assume to come in finite number. Implicit in this finite list of "possible" structures is that we can solve the non-trivial problem of identifying all dense local packings that avoid prohibitive overlap of particles. In what follows we shall assume that this problem can be addressed by simulation. An individual inherent structure of the liquid can be characterized by the frequency of each type of local structure. This introduces the structure vector $\vec{c}$ of a state of the system, where $c_{i}$ is the number concentration of particles characterized by the $i$ th local structure in that state. This vector must obey $\sum_{i} c_{i}=1$, as we assume to describe the full set of possible structures - or, at least, all structures that are likely to be observed in practice. The average structure vector $\langle\vec{c}\rangle$ over inherent structures for a given liquid corresponds to the typical data reported from structural studies of simulated liquids. The pioneering 1977 studies of Tanemura et al. 22], for example, consisted of reporting the average concentrations $\langle\vec{c}\rangle$ of Voronoi polyhedra in a soft sphere liquid.

Our key assumption, which should be valid as long as the interactions are short ranged, is that the sole data of structure concentrations, i.e. the structure vector $\vec{c}$, allows for a reasonable estimate of the energy of inherent structures. This means that the potential energy per particle of a given inherent structure of the system can be written simply as

$$
E=\vec{c} \cdot \vec{\epsilon}
$$

where $\vec{\epsilon}$ is the energy vector characterizing the local energy landscape of the system, i.e. a site with local structure $i$ contributes with an energy $\epsilon_{i}$ to the Hamiltonian. A simple choice for a vector with elements $\epsilon_{i}$ corresponding to half the average energy of a particle at the center of the in the $i$ th coordination polyhedron. Since both total energy and the $\epsilon_{i} \mathrm{~S}$ can be directly calculated, the accuracy of Eq. 2 can be tested for each specific system. Clearly this representation of the system is a simplification, as it does not include, for instance, the energy associated with distortions of local structures. From the point of view of Eq. 2, the total energy thus consists in a sum over all local structures, as previously used by Procaccia and coworkers 23] in a treatment of supercooled liquids based on the resolution of the structure into quasispecies. There is therefore no energetic coupling between neighbouring local structures: the interactions are mediated solely by the geometrical constraints arising from their overlap. This means that the identity of the coordination polyhedron of a particle will exercise a significant geometrical constraint on the possible coordination polyhedra of its neighbours. Such constraints have a purely geometrical origin, and are thus athermal.

\section{B. Configurational Entropy in Structure Space}

On cooling, the most stable local structures - those with low $\epsilon_{i}$ 's - are expected to accumulate in the liquid. This energy decrease must be balanced against the associated loss of entropy incurred by the accumulation of the stable subset of local structures. In this Section, we relate this entropy cost to the geometrical properties of these stable local structures. We have shown recently that the accumulation of high-symmetry structures is entropically penalized, as compared with low-symmetry structures 24. We now go further and encompass correlations between structures in our description.

In the high temperature limit $T>T_{x}$ where the average energy of inherent structures become temperatureindependent, it is natural to assume that their structure also reaches a limiting composition, which we denote by $\vec{c}_{\infty}=\langle\vec{c}\rangle_{T=\infty}$. We are however unaware of any examination of this proposal and whether in fact, the temperature independence of the average inherent structure energy is indeed translated into a temperature independent set of high $\mathrm{T}$ inherent structure. A true high temperature structure, under a constraint of fixed density, should correspond to the structure of purely repulsive particles, a physical limit in which the purely entropic character of the correlations become explicit.

We now consider the space formed by the possible values of $\vec{c}$ for a macroscopic system. To each such structure vector we can associate a configurational entropy

$$
S_{c}(\vec{c})=\log \Omega(\vec{c})
$$


where $\Omega(\vec{c})$ is the number of states of the liquid compatible with the macroscopic structural composition $\vec{c}$. By construction $S_{c}(\vec{c})$ is maximal at the infinite-temperature composition $\vec{c}_{\infty}$, and to second order in $\vec{c}-\vec{c}_{\infty}$ we can thus write:

$$
S_{c}(\vec{c})=S_{\infty}-\frac{1}{2}\left(\vec{c}-\vec{c}_{\infty}\right)^{T} \cdot \hat{C}^{-1} \cdot\left(\vec{c}-\vec{c}_{\infty}\right)+O\left(\left|\vec{c}-\vec{c}_{\infty}\right|^{3}\right)
$$

where $\hat{C}^{-1}$ is a positive definite matrix characteristic of the structure space of the liquid. This implies that the density of states in the structure space is approximated by a multi-dimensional Gaussian distribution,

$$
\Omega(\vec{c}) \approx \Omega_{\infty} \exp \left(-\frac{N}{2}\left(\vec{c}-\vec{c}_{\infty}\right)^{T} \cdot \hat{C}^{-1} \cdot\left(\vec{c}-\vec{c}_{\infty}\right)\right)
$$

where $N$ is the number of particles in the system. The coefficients of the matrix $\hat{C}$ thus correspond to the hightemperature covariances of the local structure concentrations, i.e.

$$
C_{i j}=N \operatorname{Cov}_{T=\infty}\left(c_{i}, c_{j}\right)
$$

In the infinite-temperature limit, structural correlations become short-ranged, and only the athermal constraints originating from the overlap of neighbouring structures are expected to contribute to the coefficients $C_{i j}$. While this property is exact in lattice models as considered in Section [II], it remains to be established in the case of off-lattice inherent structures.

\section{Thermodynamic Implications of Structural Covariance}

The connection between the structure space and the more usual microcanonical ensemble configurational entropy $S_{c}(E)$ is obtained by using Eq. 2, and integrating over structural composition to get the density of states $\Omega(E)$ as a function of the energy per particle $E$ :

$$
\Omega(E)=\int \delta(E-\vec{c} \cdot \vec{\epsilon}) \Omega(\vec{c}) \mathrm{d} \vec{c}
$$

which, employing the Gaussian approximation for the density of states introduced in Eq. 5, yields:

$$
\Omega(E)=\Omega_{\infty} \exp \left(\frac{\left(E-E_{\infty}\right)^{2}}{2 K}\right)
$$

where

$$
K=\vec{\epsilon}^{T} \cdot \hat{C} \cdot \vec{\epsilon}
$$

Note that the unit vector, $(1, \ldots, 1)$, is an eigenvector with zero eigenvalue of $\hat{C}$ (as departing from $\sum_{i} c_{i}=1$ is impossible, i.e. it has an infinite entropy cost) so that a change of energy reference does not affect the value of
$K$. Equation 8 leads us to recover the parabolic approximation for the microcanonical configurational entropy

$$
S_{c}(E)=S_{\infty}-\frac{\left(E-E_{\infty}\right)^{2}}{2 K}
$$

which takes the same form as that already introduced in Eq. 1 to phenomenologically describe simulated liquids. Finally, using Eq. 5 we can also predict how the average structural composition of the liquid evolves when the energy is lowered:

$$
\langle\vec{c}\rangle(E)=\frac{1}{\Omega(E)} \int \vec{c} \delta(E-\vec{c} \cdot \vec{\epsilon}) \Omega(\vec{c}) \mathrm{d} \vec{c}
$$

which yields:

$$
\langle\vec{c}\rangle(E)=\vec{c}_{\infty}+\frac{E-E_{\infty}}{K} \hat{C} \cdot \vec{\epsilon}
$$

This shows that the evolution of the concentration of structure $i$ depends not only on its energy $\epsilon_{i}$, but also on the energy of other structures $j$ through the geometric coupling due to their overlap:

$$
\frac{\partial\left\langle c_{i}\right\rangle}{\partial E}=\frac{1}{K} \sum_{j} C_{i j} \epsilon_{j}
$$

In particular, this shows that the accumulation of a given local structure upon cooling is not equivalent to it being stable: some possibly unfavoured structures will tend to accumulate due to their geometrical affinity to other stable local structures.

Our framework thus provides an explicit connection between the local structural properties of a liquid and its thermodynamic properties. The main outcome of our reasoning is the analytic prediction of Eq. 9 for the quadratic coefficient $K$, which is the key quantity controlling the entropic cost of lowering the potential energy of the liquid. It combines the data of the local energy landscape - the energy vector $\vec{\epsilon}$, which can be straightforwardly computed from the set of local structures - with the geometrical properties of structures the covariance matrix $\hat{C}$ encoding symmetry and overlap properties of local structures - to give a simple number that predicts the high-temperature properties of the system. Using these quantities, we are also able to predict, through Eq. 13, how the liquid's structure will change on cooling.

Note that the truncation of the expansion of the entropy to second order means that we only account for the correlations between pairs of local structures, and neglect correlations between three or more local structures. As an example of such higher order correlations neglected by this approximation, Cheng et al [25] have reported the existence of extended arrangements of local polyhedra such as rings of icosahedra. It would be in principle feasible to extend our framework to higher orders; however it would increase dramatically its complexity.

Central to our program is the calculation of the infinite-temperature structural covariance matrix $\hat{C}$, 
which encodes all pertinent information about geometrical interactions of pairs of structures. It could already be empirically obtained through Eq. 6 provided we have knowledge on the high-temperature inherent states statistics. However, we suggest that it could also be derived from first principles, using only the geometrical properties - symmetry and pair overlap - of the local structures. This program, which we have already carried on in the case of lattice liquids 26, would allow a quantitative approximation of the liquid configurational entropy in terms of purely local and geometrical properties of the liquid.

\section{APPLYING THE STRUCTURAL COVARIANCE ANALYSIS TO LATTICE SPIN MODELS}

The application of the program outlined in the previous Section to a simulated liquid with continuous degrees of freedom has not yet been carried out. In its place, we present in this Section an application of the theory to a lattice model of liquids, the Favoured Local Structure (FLS) model. Our goal is to demonstrate the applicability of the structural covariance approach to computing the configurational entropy, and investigate the range of temperatures over which it provides physically relevant results. The idea of this discrete model is that it provides a finite set of local structures, over which we can choose explicitly an arbitrary local energy landscape. We start this Section by presenting a generalized version of the FLS model, and showing how to implement our structural covariance approach within this model. We finally extend this model to the case of multiple FLS, and show how our high-temperature covariance analysis provides precious information about the glass-forming ability of the liquid.

\section{A. The Favoured Local Structures Model}

The Favoured Local Structure (FLS) model consists in two-state spins on a face-centered cubic (FCC) lattice, in which we explicitly define the Hamiltonian of the system in terms of a simple local energy landscape. Each site has 12 neighbours and on each site we have a spin that is either up or down. Among the $2^{12}=4096$ possible combinations for the spin values of these 12 neighbours, we identify 218 geometrically distinct local structures, which are not related by to one another by a rotation, as depicted in Figure 1. The principle of our model is to attribute an energy $\epsilon_{i}$ to each local structures $i$ of this set. Therefore, denoting $c_{i}$ the proportion of sites of a macroscopic system in this local structure, the average energy per site reads:

$$
E=\sum_{i=1}^{218} c_{i} \epsilon_{i}=\vec{c} \cdot \vec{\epsilon}
$$

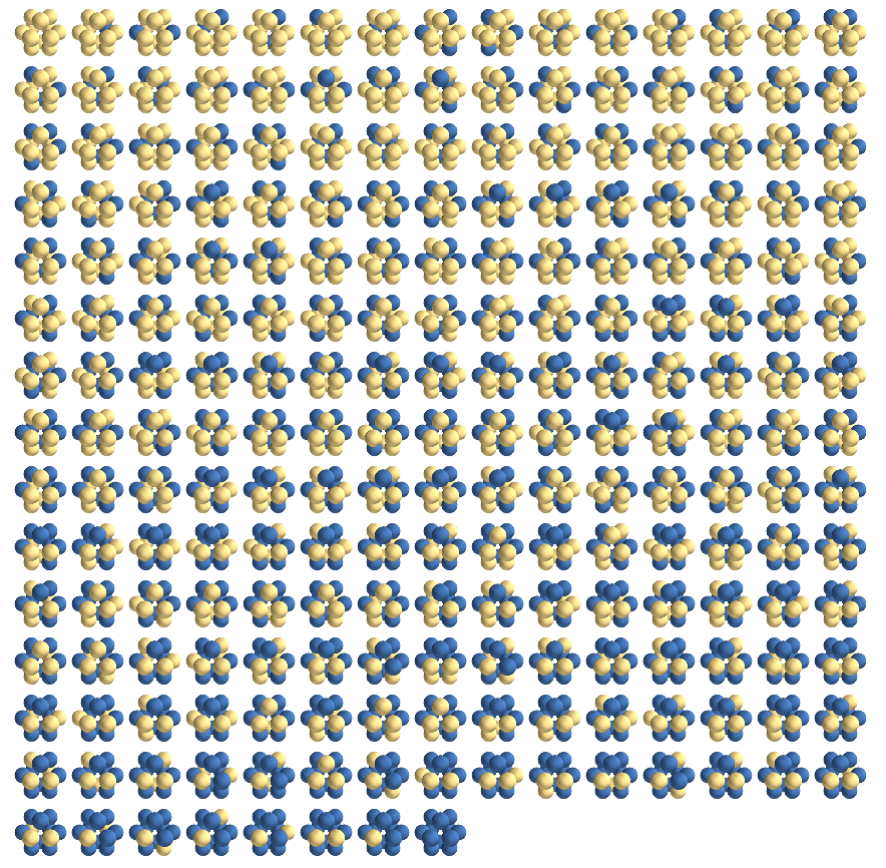

FIG. 1: The 218 local structures at the nearest neighbour level in a binary spin model on the face-centered cubic lattice, ordered by increasing number of up (blue) spins. Note that here we do not consider the value of the central spin in our description; doing so would double the number of structures.

such that our model fulfills precisely the assumptions of Section [I], where the inherent structures of the liquid are now replaced by spin configurations of the model.

An instance of the FLS model thus corresponds to the choice of the energy vector $\vec{\epsilon}$ in the huge, 218-dimensional parameter space. Note that we can select these energies arbitrarily, without requiring that they derive from a pair potential. This convenient property allows us to study directly the influence of the geometry of local structures, without being concerned with finding fine-tuned interaction potentials that would favour these structures. Once this local energy landscape $\vec{\epsilon}$ is set, Eq. 14 fully defines the Hamiltonian of the system. We typically study this model numerically, employing the Metropolis algorithm in canonical ensemble simulations of a finite system of size $30^{3}$ lattice sites, with periodic boundary conditions. Note that we do not enforce conservation of the total spin.

The aim of this model is to gain insight on the influence of locally favoured geometries on the thermodynamic properties of the system. A full study of this gigantic parameter space would be both daunting and pointless; instead, we focus on reduced versions with only a few non-zero $\epsilon_{i}$ 's. We have recently presented an extensive study [27] of the case of a single favoured local structure $i$, i.e. with $\epsilon_{j}=-\delta_{i j}$ where $\delta_{i j}$ is the discrete delta function. In that case, each site in the FLS has an energy -1 , while all others have an energy 0 , and the differences between systems can be completely attributed to 
the geometrical properties of the FLS. In particular, we showed that low-symmetry local structures tend to stabilize better the liquid than high-symmetry ones. Indeed, their entropy cost is lower due the remaining uncertainty on the orientation of low-symmetry local structures.

The other key component to the liquid's stability with respect to crystallization is the ground state energy $E_{o}$, corresponding in this model to a dense packing of FLS's on the lattice. In our model, this energy ranges from $E_{o}=-1$ (for those FLS's which can tile the whole space, such as the all-up local structure) to $E_{o}=-0.25$, corresponding to a highly frustrated local order where only one site out of four, at most, can be in the FLS, i.e. geometrical constraints prevent the local energy minimum from extending to the whole space, and effected termed geometrical frustration [28. While the connection between FLS symmetry and ground state energy turns out to be complex, we have observed that chiral FLS's - i.e. local structures lacking a plane reflection symmetry - tend to be much more frustrated than achiral ones. As we will show here, we can use this property to generate glassforming systems by combining several highly frustrated FLS's.

\section{B. Structural covariance analysis of the FLS model}

We now apply the theoretical analysis presented in Section III to this model, for any choice of the local energy landscape $\vec{\epsilon}$. Again, we first consider the entropy in the structure space $S(\vec{c})$, which is a generic property of our model, independent of $\vec{\epsilon}$.

The infinite-temperature structural composition of the liquid is simply given by the symmetry properties of local structures: each local structure $i$ can fit at any lattice site in $g_{i}=s_{i} / \omega$ distinct orientations, where $\omega=24$ is the size of the rotation group of the FCC lattice and $s_{i}$ is the cardinal of the rotation subgroup that leaves the structure unchanged. At infinite temperature, the spins are completely independent, and all $2^{12}$ local spin environments are equiprobable; hence $c_{i, \infty}=g_{i} / 2^{12}$.

Expanding the entropy $S(\vec{c})$ around the infinitetemperature composition $\vec{c}_{\infty}$ as in Eq. 4 defines the infinite-temperature covariance matrix $\hat{C}$ of structural compositions in this model (Eq. 6). This matrix is a purely geometrical property of the set of structures presented in Fig. 1. As discussed in the previous Section, this matrix provides a measure of the degree to which each possible pair of local structures act to constrain each other. The physical origin of this constraint is local, arising from the geometrical restrictions involved in the overlap of two coordination shells. In our model, this overlap consists in lattice sites being shared between adjacent local structures, as shown in Fig. 2. A complete enumeration of these local constraints allows us to compute exactly the covariance matrix $\hat{C}$, as detailed further in Ref. 26]. The principle of this calculation is to consider, for each pair of neighbouring lattice sites such that the lo-
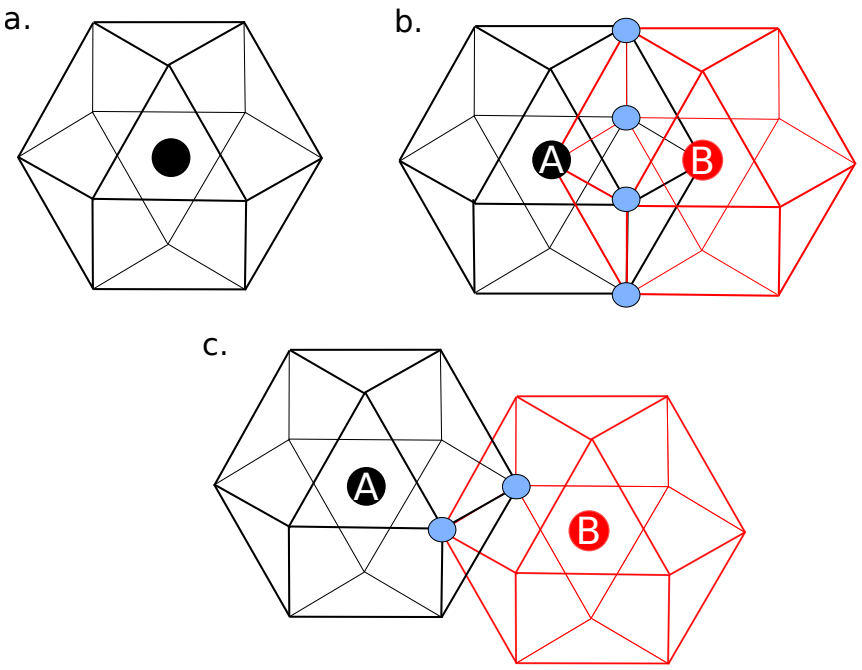

FIG. 2: (a). The local structure at a lattice site (black circle) is determined by the spin values of the sites of its coordination shell which has the geometry of a cuboctahedron. (b) and (c). The local environments of two neighbouring sites A and B overlap at 4 points (blue circles in (b)), 2 point (blue circles in (c)) or 1 point. These overlaps impose mutual constraints on the local structures that sites A and B can adopt.

cal structures overlap, the change in infinite-temperature probability of observing structure $i$ at one site knowing that structure $j$ is present at the other. If structures $i$ and $j$ are geometrically incompatible, this will be impossible and the probability will be zero, while if they are compatible the shared sites result in an enhanced probability.

In Figure 3, we present a graphical representation of the high temperature covariance matrix $\hat{C}$. We emphasize the fact that this matrix does not depend on a particular choice of the energies of the different structures (i.e. the choice of $\vec{\epsilon}$ ) as it is entirely determined by the geometrical constraints associated with overlapping coordination shells.

Combining the covariance matrix with the energy vector, we find again the quadratic approximation formula for the microcanonical entropy,

$$
S(E)=S_{\infty}-\frac{1}{2 K}\left(E-E_{\infty}\right)^{2}
$$

where the thermodynamic coefficient $K$ is given by Eq. 9. In the case of the single FLS model, with $\epsilon_{j}=-\delta_{i j}$, this coefficient becomes

$$
K=\vec{\epsilon} \cdot \hat{C} \cdot \vec{\epsilon}=C_{i i}
$$

Thus only self-overlap of the FLS is important in this quadratic approximation, while all other local structures are spectator to the accumulation of order in the liquid. In spite of its simplicity, the version of the FLS model characterized by Eq. 16 has proven useful in understanding some of the factors that influence the temperature dependence of the liquid entropy [24]. 


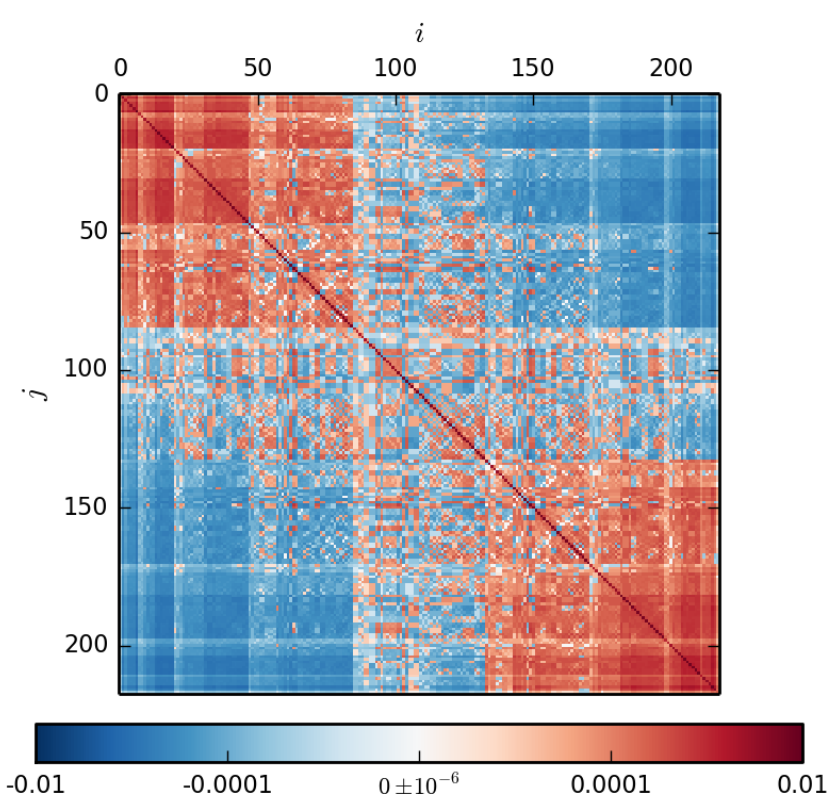

FIG. 3: A colour coded depiction of the values of the elements of the covariant matrix $\hat{C}$. Negative and positive elements are in shades of blue and red, respectively. These shades are log-scaled with a cut region around zero, as indicated by the colorbar. The local structures, listed by a numerical index running from 1 to 218 , are sorted by their number of up spins as in Figure 1 (hence the block structure of this matrix, as local structures with similar spin composition will tend to attract each other). Within each block, they are sorted with increasing geometrical multiplicity $g_{i}$.

Because in this model the total entropy equals the configurational entropy, Eq. 15 can be used to map our results to finite temperature properties through the formula $\partial S / \partial E=1 / T$. Our framework thus provides predictions for the temperature dependence of the entropy and energy of the system:

$$
\begin{aligned}
& E(T)=E_{\infty}-K / T \\
& S(T)=S_{\infty}-\frac{K}{2 T^{2}}
\end{aligned}
$$

Similarly, adapting Eq. 12 yields

$$
\langle\vec{c}\rangle(T)=\vec{c}_{\infty}-\frac{1}{T} \hat{C} \cdot \vec{\epsilon}
$$

Equations 17,19 become exact in the high-temperature limit, as they correspond to an exact expansion up to $O\left(T^{-2}\right)$. They thus provide a quantitative approximation for the liquid's thermodynamic properties and structural composition at finite temperature.

\section{Multiple FLS and Glass-Forming Ability}

We now present a new variant of this model, which has the specificity to present glass-forming systems, and use our covariance formalism to study it.

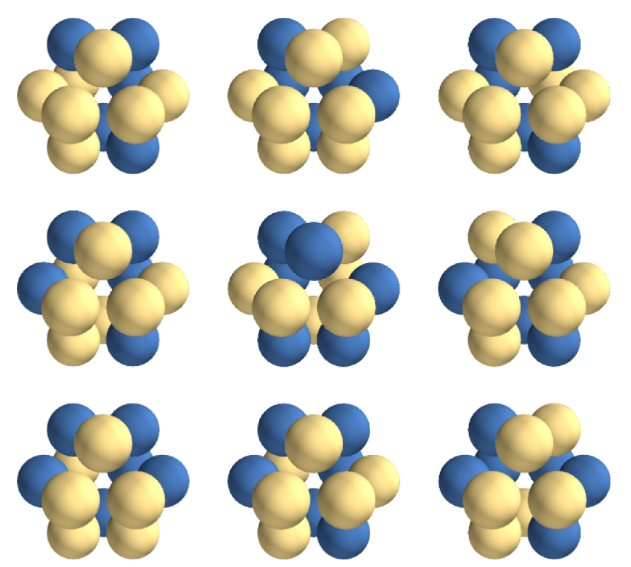

FIG. 4: Depiction of the nine FLS's we consider for our model with two favoured structures. These are denoted respectively (from left to right and top to bottom) " $43 \mathrm{c} "$, " $47 \mathrm{c}$ ", " $51 \mathrm{c} "$, "53c", "62c", "63c", "69c", "70c" and "71c" in our comprehensive study of single-FLS cases 27.

Let us consider the more general - and perhaps more realistic - scenario in which a liquid can be characterized by multiple FLS's. We model this using our lattice model by attributing an energy $\epsilon_{i}=-1$ to a number of local structures (while, again, setting the remainder to zero energy) [26]. The number of options for such liquids in the FLS model is huge - 47306 possibilities for only two FLS's in the 3D FCC model - so we shall restrict ourselves here to constructing bi-FLS liquids from a specific subset of local structures, as depicted in Fig. 4. These structures have been chosen because, in the single FLS system, they have a high ground state energy above -0.3 , meaning that at most $30 \%$ of the lattice sites can host a FLS in a given configuration of the system. This high level of frustration results in a low equilibrium freezing temperature, as the crystalline groundstate is not very stable, and therefore leads to more interesting liquid physics. Besides, the structures of Fig. 4 are chosen to have either 5 or 6 up spins, such that no ferromagnetic alignment effect takes place: only the geometrical features of the selected structures are important.

We consider here bi-FLS systems in which two of these local structures are favoured with an energy -1 , while all other structures have zero energy. Note that each of our nine selected local structures are chiral, i.e. they lack a reflection symmetry. We also include spin-inverted and mirror-symmetric cases for these structures, which have the same properties as the original FLSs, but different overlap properties with each other. This results in a pool of 126 possible pairings of such chiral, high ground state energy systems, which we have investigated comprehensively using Monte-Carlo simulations. 


\section{Scenarios for the low-temperature fate of the liquid}

We can identify two distinct scenarios in the behaviour of these liquids on cooling: they either crystallize (Figure 5), or continuously arrest into a disordered, glassy state (Figure 6).

Crystallization (79 systems). In the majority of studied bi-FLS systems, the observed scenario is the crystallization of the system on cooling. An example of this behaviour in bi-FLS systems is illustrated in Fig. 5. On cooling, the energy decreases (Figure 5a) as FLS's accumulate (Figure 5b). Freezing is marked by an abrupt first order transition in these variables. This results in the end of the liquid branch in the $S(E)$ microcanonical plot (Figure 5c). Figure 5d shows the covariance submatrix characterizing geometrical interactions between the FLS's in the system. At temperatures below the freezing point, the system is in a low energy state with periodic arrangement of both spins (Figure 5e) and the FLS sites (Figure 5:). The results of the high temperature approximation for $E(T)$, the FLS concentrations and $S(E)$ are also plotted (dotted lines). We find that the approximation increasingly underestimates the concentration of FLS and the liquid entropy as the temperature and energy, respectively, decrease. This shortcoming of the high $T$ approximation is quite generic for the FLS model and suggests the non-trivial result that the inclusion of higher order correlations between local structures serves to decrease the entropy cost of the FLS.

Glass Transition (47 systems). In more than a third of cases, crystallization is avoided, and the slow cooling of the system down to zero temperature leaves us with a dynamically arrested disordered state. We refer to this outcome as a glass transition, and illustrate it with an example bi-FLS system in Figure 6. The system continuously accumulates the two FLS's with decreasing $T$. We note that the degeneracy of these two local structures breaks down as the arrested state is approached (see Figure 6b). The temperature dependence of the energy (Figure 6a) and the variation of the entropy with energy (Figure 6c) characterise a continuous transformation into the disordered structure. A small heat capacity peak is associated with this transition which, in preliminary calculations, does not exhibit any systematic increase with system size. The absence of a dependence on system size suggests that there are no long range correlations associated with the observed glass transition. Note that among the 126 systems we studied, a few are somewhat ambiguous, as they undergo continuous accumulation of FLS's yet exhibit structured patterns that could be crystalline.

\section{Structural covariance and low-T fate of the liquid}

In these bi-FLS systems with favoured local structures $i$ and $j$, the high-temperature expansion gives a simple form for the thermodynamic constant $K$ characterizing a system:

$$
K=C_{i i}+C_{j j}+2 C_{i j}
$$

The diagonal terms $C_{i i}$ and $C_{j j}$ characterize self-overlap of the local structures [27. Structures that overlap well with themselves have larger $C_{i i}$ and accumulating them is less costly in entropy. Positive by construction, the diagonal terms are also dominated by the symmetry properties of the FLS's so that low-symmetry FLS's cost less entropy, and result in larger values of $K$.

The off-diagonal elements of the covariance matrix capture the entropic interactions between different FLS. For the examples presented in Figures 5 and 6, we present the physically relevant portion of the covariance matrices (Figures $5 \mathbf{d}$ and $6 \mathbf{d}$ ). In the crystallizing example, we have $C_{i j}=4.6 \times 10^{-5}$, indicating that the two FLS's have positive overlap, the presence of one at a given site tends to increase the probability to find the other in its surroundings. In contrast, in the glass-forming example, we have $C_{i j}=-1.6 \times 10^{-4}$, indicating an effective repulsion between local structure, the presence of one depletes the concentration of the other in the neighbourhood. This trend, $C_{i j}>0$ for crystallizers and $C_{i j}<0$ for glass formers, does not apply to every bi-FLS choice but, over our entire sample of 126 systems, is statistically significant. The average values of the off-diagonal covariance $C_{i j}$ for the crystallizers and the glass formers are, respectively,

$$
\begin{aligned}
\left\langle C_{i j}\right\rangle_{\text {crystal }} & =+0.7 \times 10^{-5} \pm 7 \times 10^{-5} \\
\left\langle C_{i j}\right\rangle_{\text {glass }} & =-3.5 \times 10^{-5} \pm 1 \times 10^{-4}
\end{aligned}
$$

Glass-forming ability therefore correlates with poor overlap between structures and hence geometric repulsion, while crystallizers are in general characterized by a geometric attraction between FLS's. This effect is statistically significant, a two-tailed Student $t$-test on the value of $C_{i j}$ for the populations of glass-formers and crystallizers indicates a $p$-value of $p=0.007$. This correlation has however a rather low signal-to-noise ratio, as shown in Figure 3, where the distributions of the structural covariances overlap between the two populations. Although the correlation is weak, the fact that a discernable correlation exists is significant, as it supports our proposal that the high $T$ structural correlations contains information about the fate of the low $T$ liquid.

\section{The role of crystal groundstate energies}

While we have established that the high $T$ covariance between FLS's contains information relevant to the lowtemperature fate of a liquid, the weakness of the correlation indicates that higher order entropic interactions between FLS, missing from the high $T$ expression, are important. One accessible source of information on the organization of FLS at low $T$ is the crystal groundstate. This state represents the densest packing of the 

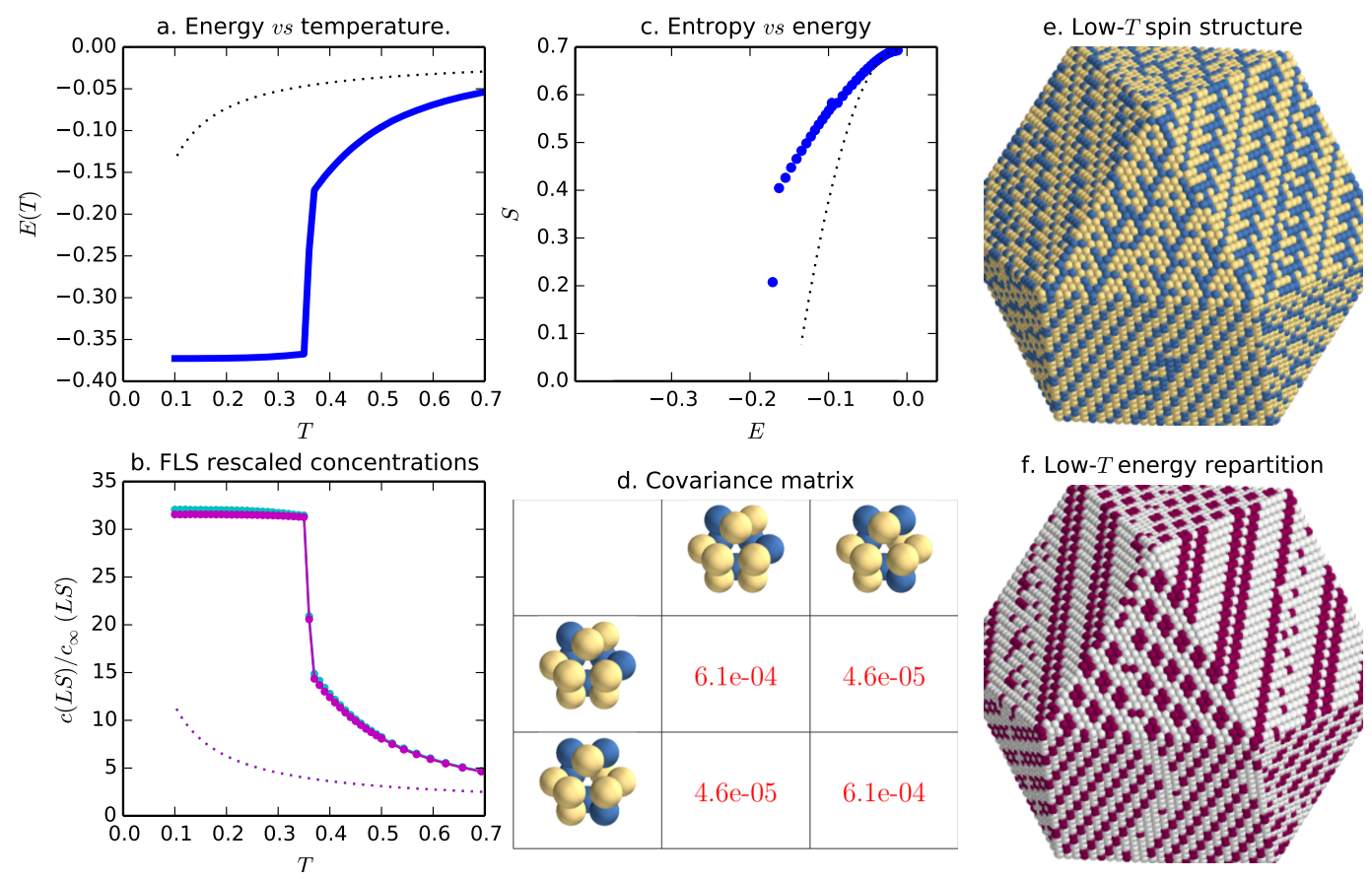

FIG. 5: An example of a bi-FLS model that crystallizes. Structures $47 c$ and $51 c$ are both favoured. a. The average energy per site exhibits a sharp first-order phase transition on cooling. Dotted line: the high- $T$ expansion prediction (Eq. 17). b. The concentration of the two FLS's (filled circles of purple and blue) as a function of temperature. The concentrations predicted by the high $T$ approximation (i.e. Eq. 19) are also included (dotted line) for comparison. c. The liquid entropy per site as a function of the energy. Also plotted, for comparison, is the high- $T$ quadratic form (Eq. 15, dotted line) which becomes exact near $E_{\infty}$. d. The two favoured local structures and their associate high- $T$ covariance. The positive off-diagonal terms indicate geometric affinity between these structures. e. Slices of a low-temperature configuration reveal a crystalline structure. f. The loci of FLS (red spheres) are regularly organized. Parameters: system size $30 \times 30 \times 30$ with periodic boundary conditions; cooling rate $10^{6}$ Monte-Carlo steps/site/temperature unit.

selected FLS on the FCC lattice. Establishing the crystal groundstates for 126 different Hamiltonians via structural searches is a substantial challenge. We have applied the lattice search algorithm described in ref. [27, an enumerative method encompassing all possible crystalline structures with crystal cell size $\mathcal{Z} \leq 64$ and complemented by large-scale Monte-Carlo simulations. Since the same overlap constraints in the bi-FLS models influence the groundstate energy and the high $T$ covariance $C_{i j}$ we would expect some correlation between the two quantities. We test this conjecture in Figure 9 where we plot the scatter of the pairs $\left(C_{i j}, E_{o}\right)$ for all 126 systems studied. We find a negative correlation between $E_{o}$ and $C_{i j}$, with Pearson correlation coefficient $r=-0.34$ and significance $p=10^{-4}$. Again, this correlation is rather weak, but significant. High-temperature covariances correspond to simple combinatorics that do not encompass the whole complexity of dense packing problems but they serve as useful indicators of whether FLS's will pack well or not.

$E_{o}$ also correlates with glass-forming ability. High ground state energies result in stabilization of the liquid with respect to crystallization and, therefore, in low the crystallization temperatures. This in turn increases the likelihood that the liquid will dynamically arrest be- fore freezing. Averaging the ground state energies with crystallizing and glass-forming systems, we find:

$$
\begin{aligned}
\left\langle E_{o}\right\rangle_{\text {crystal }} & =-0.355 \pm 0.04 \\
\left\langle E_{o}\right\rangle_{\text {glass }} & =-0.327 \pm 0.02
\end{aligned}
$$

i.e., the former have significantly lower ground state energies than the latter. A Student $t$-test indicates a strong significance for this trend, with $p=10^{-6}$. We thus conclude that a possible mechanism explaining why geometric repulsion between favoured local structures correlates with glass-forming ability is through the high ground state energy it incurs.

\section{What determines whether a liquid will crystallize or form a glass?}

The question of what factors influence the fate of the liquid is of central importance both for the development of new bulk metallic glasses [29] and in terms of understanding the fundamental question of the low temperature end of the liquid state. The kinetics of crystallization is obviously of central importance. The relationship between crystal nucleation and growth and the structural 

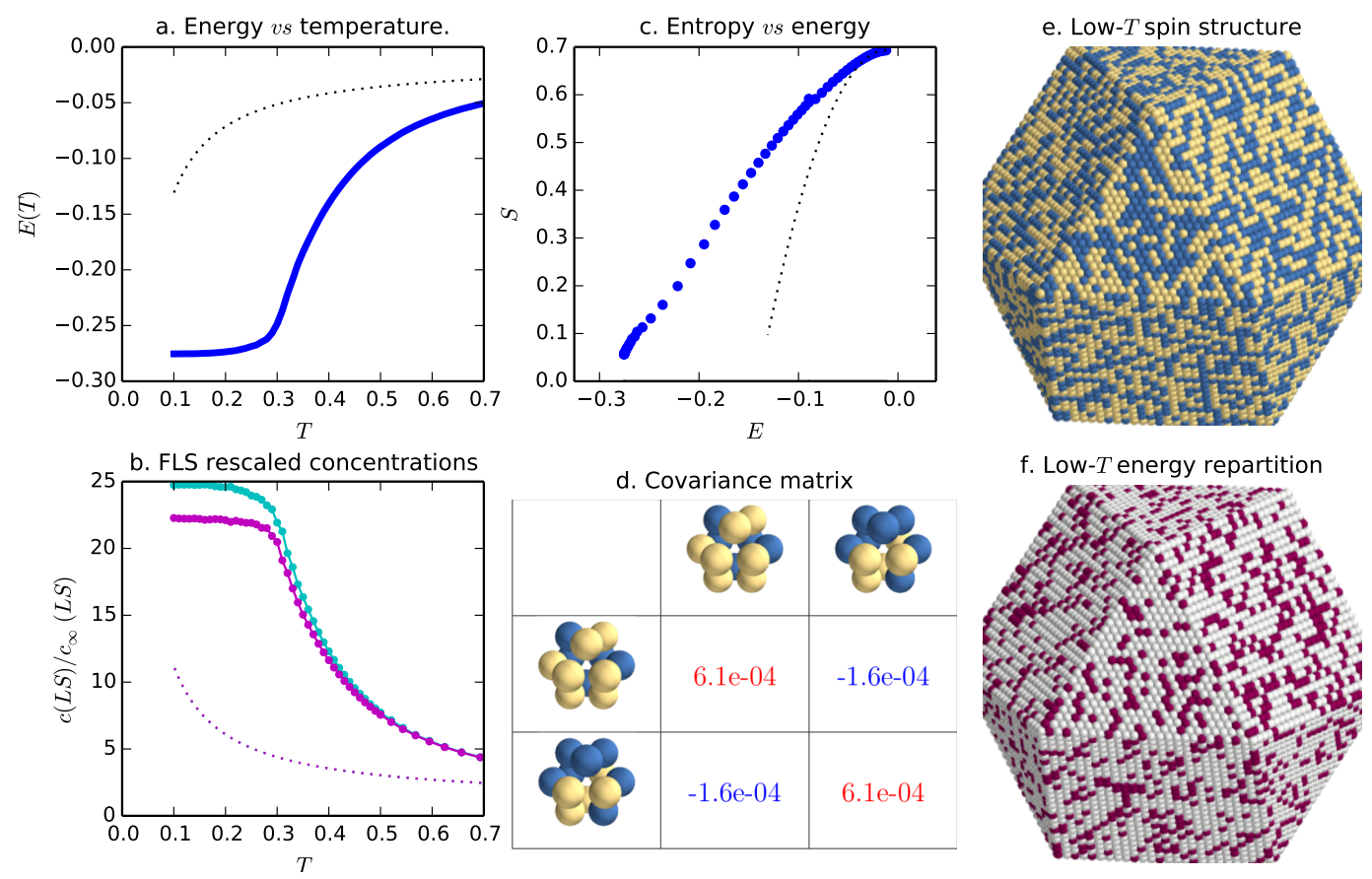

FIG. 6: An example of a glass-forming bi-FLS system: structures $47 c$ and the spin-inverted, mirror-symmetric version of $51 c$ are both favoured. a. The average energy per site exhibits a continuous decrease on cooling, down to the point of dynamical arrest. Dotted line: the high-T expansion prediction (Eq. 17). b. The concentrations of the two FLS's (filled circles of purple and blue) as a function of temperature. c. The liquid entropy per site as a function of the energy. Also plotted, for comparison, is the high- $T$ quadratic form (Eq. 15 dotted line). d. The two favoured local structures and their associate high- $T$ covariance. The negative off-diagonal terms indicate geometric repulsion between these structures. e. Slices of a low-temperature configuration reveal an absence of crystallinity. f. The loci of FLS (red spheres) are apparently random. Parameters: system size $30 \times 30 \times 30$ with periodic boundary conditions; cooling rate $10^{6}$ Monte-Carlo steps/site/temperature unit.

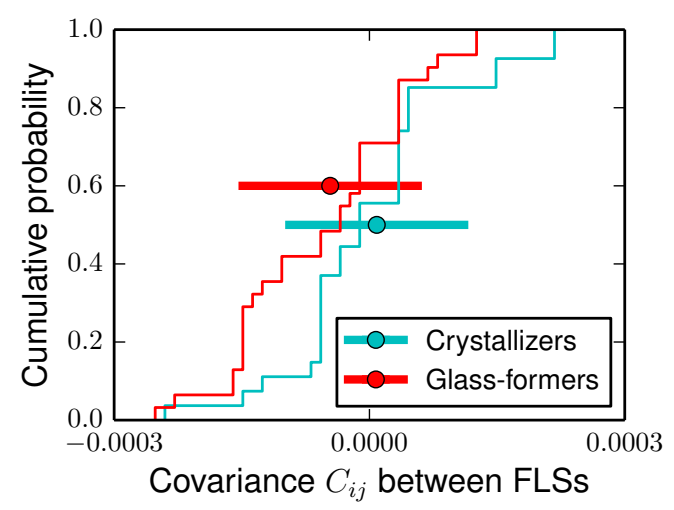

FIG. 7: The cumulative probability of the structural covariances, for the 47 glass-forming and the 79 crystallizing systems. The filled circles with error bars indicate the mean and standard deviation, respectively, within each population.

statistics of the liquid is a substantial problem that we leave for future work. Here we restrict ourselves to consider a key parameter of the crystallization kinetics: the equilibrium freezing temperature. The lower this temperature, the slower the relaxation of the system at the freezing point, and hence the higher the likelihood to miss

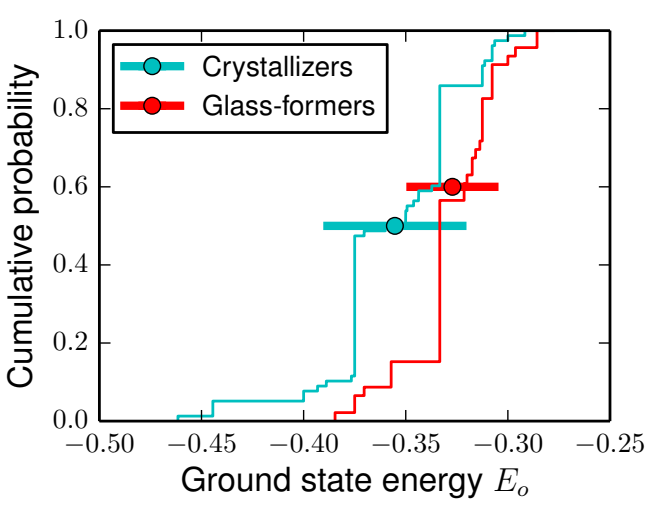

FIG. 8: The cumulative probability of the ground state energies $E_{o}$. The filled circles with error bars indicate the mean and standard deviation, respectively, within each population.

crystallization and form a glass.

Previously [26, we derived an expression for the freezing temperature $T_{f}$ based on the high $T$ expression for the entropy,

$$
T_{f}=\left(\frac{E_{\infty}-E_{o}}{2 S_{\infty}}\right)\left(1+\sqrt{1-\frac{2 K S_{\infty}}{\left(E_{\infty}-E_{o}\right)^{2}}}\right)
$$




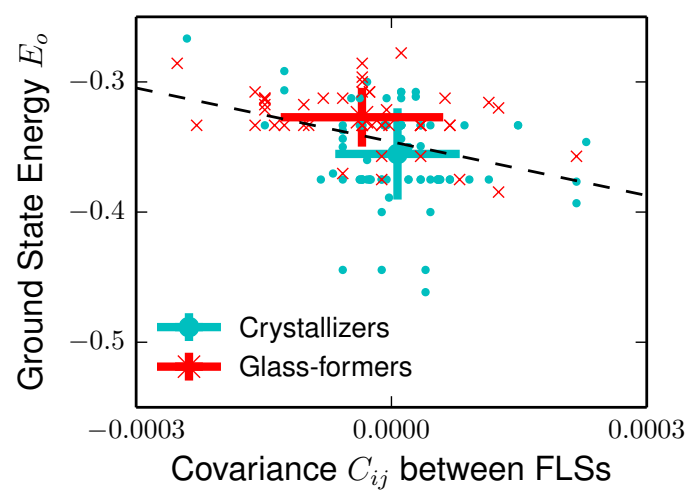

FIG. 9: Scatter plot of the values of the ground state energy $E_{o}$ as a function of structural covariance $C_{i j}$ for all 126 bi-FLS systems. Dots: crystallizing systems, crosses: glass-forming systems. The large symbols with error bars indicate the mean and standard deviations within each population. The black dashed line is a linear regression.

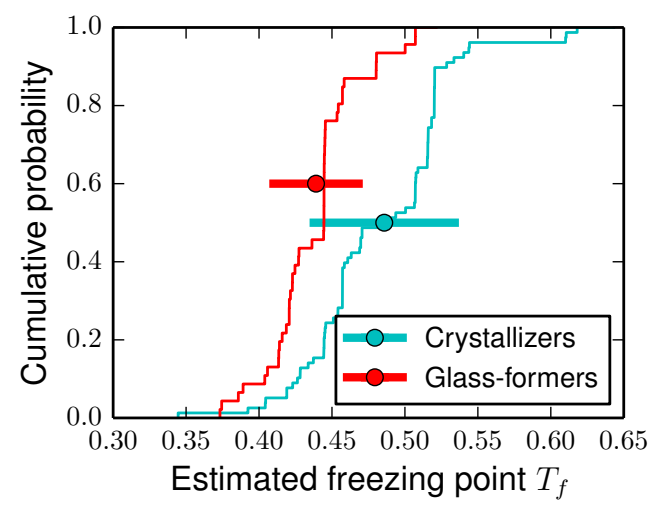

FIG. 10: The cumulative probability of the ground state energies $E_{o}$. The filled circles with error bars indicate the mean and standard deviation, respectively, within each population.

Here $E_{\infty}$ and $S_{\infty}$ are the high $T$ limit of the energy and entropy per site, respectively. In Figure 10 we plot the cumulative distributions of this estimated value for $T_{f}$ for the glass forming and crystallizing bi-FLS models. While the two distributions still overlap, they appear to be better separated by $T_{f}$. Quantitatively, we find:

$$
\begin{aligned}
\left\langle T_{f}\right\rangle_{\text {crystal }} & =0.49 \pm 0.05 \\
\left\langle T_{f}\right\rangle_{\text {glass }} & =0.44 \pm 0.03
\end{aligned}
$$

and the $p$-value is now $p=10^{-7}$, lower than for both $C_{i j}$ and $E_{o}$. By combining information of both the high $T$ structural correlations and the $T=0$ the groundstate energy $E_{o}$ into an estimated freezing temperature, we thus obtain a better predictor of the glass-forming ability of a system than by the covariance $C_{i j}$ alone. This result is consistent with the experimental observation 30 that a low freezing point is one of the best indicators of glass forming ability.

\section{DISCUSSION}

In this article, we have proposed a general framework for analysing the statistics of liquid structure and connecting explicitly these correlations to the configurational entropy of the liquid. We have argued that the fluctuation statistics of the high temperature liquid (or, equivalently, a version of the liquid with only repulsions retained) contain important information on the geometrical constraints of the liquid, even in the supercooled regime. This proposal runs counter to the current practice in computational studies of liquid structure to look in the lowest temperature liquids, an approach ideally suited to identify what structures are present but unhelpful in addressing why these structures are so favoured. We have addressed this why question with an explicit expression for the configurational entropy in terms of the statistics of the local structures, revealing in particular the importance of the covariance of the structural fluctuations. With our new approach to the analysis of liquid structure, we have thus presented in this article a first step towards a structural characterization of amorphous configurations that goes beyond merely descriptive approaches.

As a testing ground for our theory, we have considered a large sample of model liquids within our Favoured Local Structure lattice model. Here, each liquid we consider is characterised by two FLSs drawn from a selection of low-symmetry structures. A modest correlation between glass forming ability and the high $T$ covariance $C_{i j}$ serves both to support our claims for the relevance of the high temperature structural statistics. Beyond providing a glimpse on the mechanisms leading to a glass transition, we note that this model also gives us a reliable way to generate glass-forming systems in discrete lattice models without spatial heterogeneity or frozen disorder. Such a simple geometrical model may prove useful to address the challenges that remain in explaining the physics of the glass transition.

\section{ACKNOWLEDGEMENTS}

Acknowledgements $\mathrm{PH}$ acknowledges the financial support of the Australian Research Council. PR is supported by "Initiative Doctorale Interdisciplinaire 2013" from IDEX Paris-Saclay.
[1] J.-P. Hansen and I. R. Macdonald, Theory of Simple Liquids (Academic Press, London, 1990)
[2] V. K. De Souza and P. Harrowell, Phys. Rev. E 80, 
041503 (2009).

[3] S. Saw and P. Harrowell, Phys. Rev. Lett. 116, 137801 (2016).

[4] G. Wahnström, Phys. Rev. A 44, 3752 (1991).

[5] G. M. Hocky, D. Coslovich, A. Ikeda and D. R. Reichman, Phys. Rev. Lett. 113, 157801 (2014); A. J. Dunleavy, K. Wiesner, R. Yamamoto and C. P. Royall, Nat. Comm.6, 6089 (2015).

[6] P. G. Debenedetti and F. H. Stillinger, Nature 410, 259 (2001).

[7] F. Sciortino, W. Kob and P. Tartaglia, Phys. Rev. Lett. 83, 3214 (1999).

[8] S. Büchner and A. Heuer, Phys. Rev. E 60, 6507 (1999).

[9] I. Saika-Voivod, P. H. Poole and F. Sciortino, Nature 412, 514 (2001); ibid, Phys. Rev. E 69, 041503 (2004); A. Saksaengwijit, J. Reinisch and A. Heuer, Phys. Rev. Lett. 95, 235701 (2004).

[10] A. J. Moreno, A. Saika-Voivod, E.Zaccarelli, E. La Nave, S. V. Buldyrev, P. Tartaglia and F. Sciortino, J. Chem. Phys. 124, 204509 (2006).

[11] S. Sastry, J. Phys.: Cond. Matt. 12, 6515 (2000); B. Doliwa and A. Heuer, Phys. Rev. E 67, 031506 (2003).

[12] A. Saksaengwijit, J. Reinisch and A. Heuer, Phys. Rev. Lett. 93, 235701 (2004).

[13] S. Sastry, P. G. Debenedetti and F. H. Stillinger, Nature 393, 554 (1998)

[14] J. D. Bernal, Nature 183, 141 (1959); A. Rahman, J. Chem. Phys. 45, 2585 (1966); J. L. Finney, Proc. Roy, Soc. A 319479 (1970).

[15] T. Ogawa and M. Tanemura, Proc. Theor. Phys. 51, 399 (1974); M. Tanemura, Y. Hiwatari, H. Matsuda, T. Ogawa, N. Ogita and A. Ueda, Prog. Theor. Phys. 58, 1079 (1977).

[16] H. Jónsson and H. C. Andersen, Phys. Rev. Lett. 602295 (1988).
[17] Y. H. Chui, R. J. Rees, I. K. Snook, B. OMalley and S. P. Russo, J. Chem. Phys. 125, 114703 (2006).

[18] P. J. Steinhardt, D. R. Nelson and M. Ronchetti, Phys. Rev. B 28, 784 (1983).

[19] J. P. K. Doye and D. J. Wales, Phys. Rev. Lett. 86, 5719 (2001).

[20] C. P. Royall and S. R. William, Phys. Rep. 560, 1 (2015); Y. Q. Cheng and E. Ma, Prog. Mater. Sci. 56, 379 (2011).

[21] F. C. Frank and J. S. Kasper, Acta Crystallogr. 11, 184 (1958).

[22] M. Tanemura, Y. Hiwatari, H. Matsuda, T. Ogawa, N. Ogita and A. Ueda, Prog. Thero. Phys. 58, 1079 (1977).

[23] I. Procaccia, Eur. Phys. J. Special Topics 178, 81 (2009); H. G. E. Hentschel, V. Ilyin, N. Makedonska, I. Procaccia and N. Schupper, Phys. Rev. E 75, 050404 (2007); L. Boué, E. Lerner, I. Procaccia and J. Zylberg, J. Stat. Mech. P11010 (2009); L. Boué, H. G. E. Hentschel, V. Ilyin and I. Procaccia, J. Phys. Chem. B 115, 14301 (2011); I. Procaccia and I. Regev, Europhys. Lett. 97, 36010 (2012).

[24] P. Ronceray and P. Harrowell, J. Chem. Phys. 136, 134504 (2012).

[25] Y. Q. Cheng, H. W. Sheng and E. Ma, Phys. Rev. B 78, 014207 (2008).

[26] P. Ronceray and P. Harrowell, arXiv:1510.03073 (2016).

[27] P. Ronceray and P. Harrowell, Soft Matt. 11, 3322 (2015).

[28] G. Tarjus, S. A. Kivelson, Z. Nussinov and P. Viot, J. Phys.: Cond. Matt. 17, R1143 (2005).

[29] W. H. Wang, C. Dong and C. H. Shek, Mater. Sci. Eng. Rep. 44, 45 (2004).

[30] D. Turnbull,Contemp. Phys. 10, 473 (1969); W. Ping, D. Paraska, R. Baker, P. Harrowell and C. A. Angell, J. Phys. Chem. B 115, 4696 (2011). 